\title{
A novel fiber laser development for photoacoustic microscopy
}

\author{
Seydi Yavas ${ }^{a}$, Esra Aytac-Kipergil ${ }^{b}$, Mustafa U. Arabul ${ }^{c}$, Hakan Erkol ${ }^{b}$, Onder Akcaalan ${ }^{a}$, \\ Y. Burak Eldeniz ${ }^{e}$ F. Omer Ilday ${ }^{a}$ and Mehmet B. Unlu ${ }^{b, d}$ \\ ${ }^{a}$ Department of Physics, Bilkent University, Bilkent, 06800, Ankara, Turkey; \\ ${ }^{b}$ Department of Physics, Bogazici University, Bebek, 34342, Istanbul, Turkey; \\ ${ }^{c}$ Institute of Biomedical Engineering, Bogazici University, Kandilli, 34684, Istanbul, Turkey; \\ ${ }^{d}$ Center for Life Sciences and Technologies, Bogazici University, Kandilli, 34342, Istanbul, \\ Turkey \\ ${ }^{e}$ Department of Electrical and Electronics Engineering, Ankara University, Tandogan, 06100, \\ Ankara, Turkey
}

\begin{abstract}
Photoacoustic microscopy, as an imaging modality, has shown promising results in imaging angiogenesis and cutaneous malignancies like melanoma, revealing systemic diseases including diabetes, hypertension, tracing drug efficiency and assessment of therapy, monitoring healing processes such as wound cicatrization, brain imaging and mapping. Clinically, photoacoustic microscopy is emerging as a capable diagnostic tool. Parameters of lasers used in photoacoustic microscopy, particularly, pulse duration, energy, pulse repetition frequency, and pulse-to-pulse stability affect signal amplitude and quality, data acquisition speed and indirectly, spatial resolution. Lasers used in photoacoustic microscopy are typically Q-switched lasers, low-power laser diodes, and recently, fiber lasers. Significantly, the key parameters cannot be adjusted independently of each other, whereas microvasculature and cellular imaging, e.g., have different requirements. Here, we report an integrated fiber laser system producing nanosecond pulses, covering the spectrum from $600 \mathrm{~nm}$ to $1100 \mathrm{~nm}$, developed specifically for photoacoustic excitation. The system comprises of Yb-doped fiber oscillator and amplifier, an acousto-optic modulator and a photonic-crystal fiber to generate supercontinuum. Complete control over the pulse train, including generation of non-uniform pulse trains, is achieved via the AOM through custom-developed field-programmable gate-array electronics. The system is unique in that all the important parameters are adjustable: pulse duration in the range of 1-3 ns, pulse energy up to $10 \mu \mathrm{J}$, repetition rate from $50 \mathrm{kHz}$ to $3 \mathrm{MHz}$. Different photocoustic imaging probes can be excited with the ultrabroad spectrum. The entire system is fiber-integrated; guided-beam-propagation rendersit misalignment free and largely immune to mechanical perturbations. The laser is robust, low-cost and built using readily available components.
\end{abstract}

Keywords: Photoacoustic imaging, fiber laser, mode-locked, supercontinuum, photonic crystal fiber

\section{INTRODUCTION}

Pulsed lasers have critical effects on quality of photoacoustic imaging, especially for life science applications. In particular, pulsed lasers with durations range from 1 ns to $50 \mathrm{~ns}$ are used for photoacoustic imaging systems. Nanosecond pulses mostly rely on Q-switched lasers, whereas, some may be generated by directly modulated diode lasers. Yet, their pulse repetition frequency (PRF) are on the order of $\mathrm{Hz}$ and rarely on the order of $\mathrm{kHz}$. Beyond $\mathrm{MHz}$ region is harder to be approached for Q-switch and modulated diode lasers.

In literature, Wang et al. used $1064 \mathrm{~nm}$ fiber laser with $10 \mathrm{~ns}$ pulse width and $50 \mathrm{kHz}$ PRF. ${ }^{1}$ Shi et al. used Q-switched microchip and fiber laser in $537 \mathrm{~nm}$ and achieved $250 \mathrm{~ns}$ pulses with $100 \mathrm{kHz}$ PRF. ${ }^{2}$ In 2011, Shi et al. used diode-pumped nanosecond-pulsed ytterbium-doped $532 \mathrm{~nm}$ fiber laser with pulse repetition rate up

Seydi Yavas: E-mail: seydi@bilkent.edu.tr, Telephone: +90 3122908070

Esra Aytac-Kipergil: E-mail: esra.aytac@boun.edu.tr, Telephone: +90 2123594782

Mehmet B. Unlu: E-mail: burcin.unlu@boun.edu.tr, Telephone: +902123596885

Photons Plus Ultrasound: Imaging and Sensing 2013, edited by Alexander A. Oraevsky, Lihong V. Wang, Proc. of SPIE Vol. 8581, 85813S - (C) 2013 SPIE · CCC code: 1605-7422/13/\$18 · doi: 10.1117/12.2004910 
to $600 \mathrm{kHz}$ in photoacoustic microscopy system. ${ }^{3}$ In 2012, Zhang et al. used two lasers for imaging myocardial sheet architecture of a mouse. They used an integrated diode-pumped Q-switched Nd:YAG laser with a tunable wavelength between 210 to $2600 \mathrm{~nm}$ and $1 \mathrm{kHz}$ PRF. For faster data acquisition, they also used a Nd:YVO4 laser with a fixed wavelength of $532 \mathrm{~nm}$ providing $50 \mathrm{kHz}$ repetition rate. ${ }^{4}$

Mode-locked lasers generally output sub-nanosecond pulses with few $\mathrm{MHz}$ repetition frequencies. In order to get nanosecond pulses with mode-locked laser, one should precisely design pulse picking systems, pulse stretchers and compressors. All the dynamics of mode-locked lasers, such as Kerr nonlinearity, dispersion, and gain have major roles in determining pulse duration and repetition frequency of laser. In a frequently appearing mode of laser, very long and incompressible pulses are generated. Although, that mode is generally disregarded, use of non-linear loop mirrors (NOLM) as saturable absorbers (SA) with sinusoidal transmission might create shorter pulses. If pump power exceeds a critical threshold, the pulse will split into a bunch of narrower pulses due to peak power clamping effect. The whole process provides pulse train with few nanoseconds pulse duration and $\mathrm{MHz}$ pulse repetition frequency.

In this study, we demonstrate a custom made fiber laser, which has 1-3 ns adjustable pulse duration, $50 \mathrm{kHz}$ to $3 \mathrm{MHz}$ selectable PRF, $600 \mathrm{~nm}$ to $1100 \mathrm{~nm}$ supercontinuum bandwidth , maximum pulse energy $10 \mu \mathrm{J}$. This unique laser is particularly designed for multispectral photoacoustic microscopy. The use of NOLM in producing nanosecond pulses is inspired of the high power material processing lasers. ${ }^{5}$

\section{EXPERIMENT SETUP}

Schematic of the experimental setup can be seen in Figure 1. The oscillator consists of a $0.7 \mathrm{~m}$ long Yb-doped fiber, followed by a $70 \%$ coupler, an $8 \mathrm{~nm}$ bandpass filter, an inline isolator to ensure unidirectional operation, an output coupler of 10\%, two polarization controllers, a $50 \mathrm{~m}$ long HI-1060 single mode fiber for Sagnac loop, and $2 \times 240 \%$ coupler for NOLM. ${ }^{5,6}$ As a pump source, a fiber-coupled single mode $980 \mathrm{~nm}$ diode laser delivering a maximum power of $650 \mathrm{~mW}$ is used. Pump protection filter rejects undesired backward propagating light within the fiber, if any, because backward power can cause laser diode to break down. The fundamental repetition rate of the cavity is 3.1 MHz. Polarization controllers are used manually in order to assist mode-locked operation of the laser. Output is split with a $10 \%$ fiber which seeds the amplification stage with oscillations of $24 \mathrm{~mW}$ power and $8 \mathrm{~nJ}$ pulse energy. Preamplifier of two stage all-fiber amplifier, comprises of $1.0 \mathrm{~m}$ long Yd doped fiber followed by fiber-integrated acousto-optic modulator (AOM) that is connected to field-programmable gate-array (FPGA). Fiber-coupled single-mode $980 \mathrm{~nm}$ diode laser delivering a maximum power of $650 \mathrm{~mW}$ is used as pump source. The AOM allows the frequency, intensity and direction of laser beam to be modified. The amplifier levels the signal up to $300 \mathrm{~mW}$ power that corresponds to $100 \mathrm{~nJ}$ energy. Complete control over the pulse train, including generation of non-uniform pulse trains, is achieved via the AOM through FPGA. A secondary amplifier including $980 \mathrm{~nm}$ diode laser delivering a maximum power of $650 \mathrm{~mW}$ and $1,0 \mathrm{~m}$ long Yd doped fiber is built in order to compensate for decreased optical power, and average power is increased to $150 \mathrm{~mW}$ that corresponds to 1.5 $\mu \mathrm{J}$ pulse energy. Pulse repetition frequency of the laser can be adjusted between $50 \mathrm{kHz}$ and $3.1 \mathrm{MHz}$ via the FPGA circuit. The temporal profile of the pulses are measured with a $45 \mathrm{GHz}$ sampling oscilloscope and $15 \mathrm{GHz}$ photodiode, with a combined rise time of 75 ps. For final amplifier, a $105 \mu \mathrm{m}$ core multimode fiber coupled 976 $\mathrm{nm}$ diode laser of delivering a maximum power of $8 \mathrm{~W}$ is used as a pump source. The amplifier also includes 2 $\mathrm{m}$ long wide band double-clad Yb-doped fiber with $20 \mu \mathrm{m}$ core diameter and numerical aperture of 0.07 . The octagonal clayed fiber of the power amplifier has $125 \mu \mathrm{m}$ core diameter and numerical aperture of 0.46 . The protection of the preamplifier is ensured by high-power isolator. If a diode laser of $25 \mathrm{~W}$ is used in the final amplifier, the optical power can be increased up to $3.5 \mathrm{~W}$. Figure 2 shows the optical spectrum and Figure 3 illustrates temporal profile of the laser before supercontinuum generation. The amplifier output before PCF reaches $2.8 \mathrm{~W}$ at $50 \mathrm{kHz}, 3.15 \mathrm{~W}$ at $100 \mathrm{kHz}$ and $3.48 \mathrm{~W}$ at $1 \mathrm{MHz}$ repetition frequency. A $3.5 \mathrm{~m}$ long photonic crystal fiber (PCF) is spliced to double-clad Yb-doped fiber of the final amplifier for supercontinuum generation. The power delivery between wide band fiber and PCF is around $35 \%$. Considering $50 \%$ beamsplitting, the output power reaches $600 \mathrm{~mW}$ which correspons to $6 \mu \mathrm{J}$ pulse energy. PCF enhances nonlinear optical propagation due to its air silica honeycomb-like microstructure. ${ }^{7}$ The zero dispersion wavelength of the PCF is $1040 \mathrm{~nm}$. As a result of non-linear effects, the spectrum broadens and supercontinuum between 600-1100 nm as seen in Figure 4 is generated. The wavelength window covers absorption maxima of tissue chromophores such as oxygenated 
hemoglobin ( $\mathrm{HbO} 2)$, deoxygenated hemoglobin ( $\mathrm{HHb})$, water, lipid, melanin, collagen and elastin. ${ }^{8}$ Filtering is required to excite different probes of interest, adjusting the central wavelength, AOM can be used as band filter for the purpose. Wavelength selection is also possible with optical filters of several wavelengths. By means of optical filters, probes can also be exposed to multiple wavelengths.

Although, some lasers in the market have tunability over wavelength on the expense of energy, repetition frequency and pulse energy are usually constant. As a result of the constraints, lasers are chosen regarding the application. A laser suitable for melanoma cell detection with a wavelength of $1064 \mathrm{~nm}$, e.g., is not appropriate for hemoglobin imaging since at the particular wavelength the absorption of hemoglobin is very low. Another laser with tunable wavelength may not be used for real time imaging because of having low PRF. Lasers having control over PRF, usually fiber lasers, have fixed wavelengths which is not suitable for spectroscopic measurements. Fixed energy obligates to perform microvasculature or cellular imaging. The laser designed specifically for photoacoustics gives freedom to select wavelength between $600 \mathrm{~nm}$ and $1100 \mathrm{~nm}$, energy between $2 \mathrm{~nJ}$ to $10 \mu \mathrm{J}$, and repetition frequency between $50 \mathrm{kHz}$ to $3 \mathrm{MHz}$; thus allows single laser utilization for various applications.

\section{ACKNOWLEDGEMENTS}

This research is supported in part by Marie Curie Reintegration Grant 268287, The Scientific and Technological Research Council of Turkey (TUBITAK) 112T253 Project, Bogazici University Research funding BAP 6033 and BAP 6190 .

\section{REFERENCES}

[1] Wang, Y., Maslov, K., Zhang, Y., Hu, S., Yang, L., Xia, Y., Liu, J., and Wang, L. V., "Fiber-laser-based photoacoustic microscopy and melanoma cell detection," Journal of Biomedical Optics 16(1), 011014-0110144 (2011).

[2] Shi, W., Kerr, S., Utkin, I., Ranasinghesagara, J., Pan, L., Godwal, Y., Zemp, R. J., and Fedosejevs, R., "Optical resolution photoacoustic microscopy using novel high-repetition-rate passively q-switched microchip and fiber lasers," Journal of Biomedical Optics 15(5), 056017-056017-7 (2010).

[3] Shi, W., Hajireza, P., Shao, P., Forbrich, A., and Zemp, R., "In vivo near-realtime volumetric opticalresolution photoacoustic microscopy using a high-repetition-rate nanosecond fiber-laser.," Opt Express 19(18), 17143-50 (2011).

[4] Zhang, C., Cheng, Y.-J., Chen, J., Wickline, S., and Wang, L. V., "Label-free photoacoustic microscopy of myocardial sheet architecture," Journal of Biomedical Optics 17(6), 060506-1-060506-3 (2012).

[5] Özgören, K., Öktem, B., Yilmaz, S., Ömer Ilday, F., and Eken, K., "83 w, 3.1 mhz, square-shaped, 1 ns-pulsed all-fiber-integrated laser for micromachining," Opt. Express 19(18), 17647-17652 (2011).

[6] Doran, N. J. and Wood, D., "Nonlinear-optical loop mirror," Opt. Lett. 13(1), 56-58 (1988).

[7] Liu, M. and Buma, T., "Wavelength agile photoacoustic microscopy with a pulsed supercontinuum source," in [IEEE Ultrasonics Symposium (IUS)], $382-385$ (2010).

[8] Beard, P., "Biomedical photoacoustic imaging," Interface Focus 1(4), 602-631 (2011). 


\section{FIGURES AND TABLES}

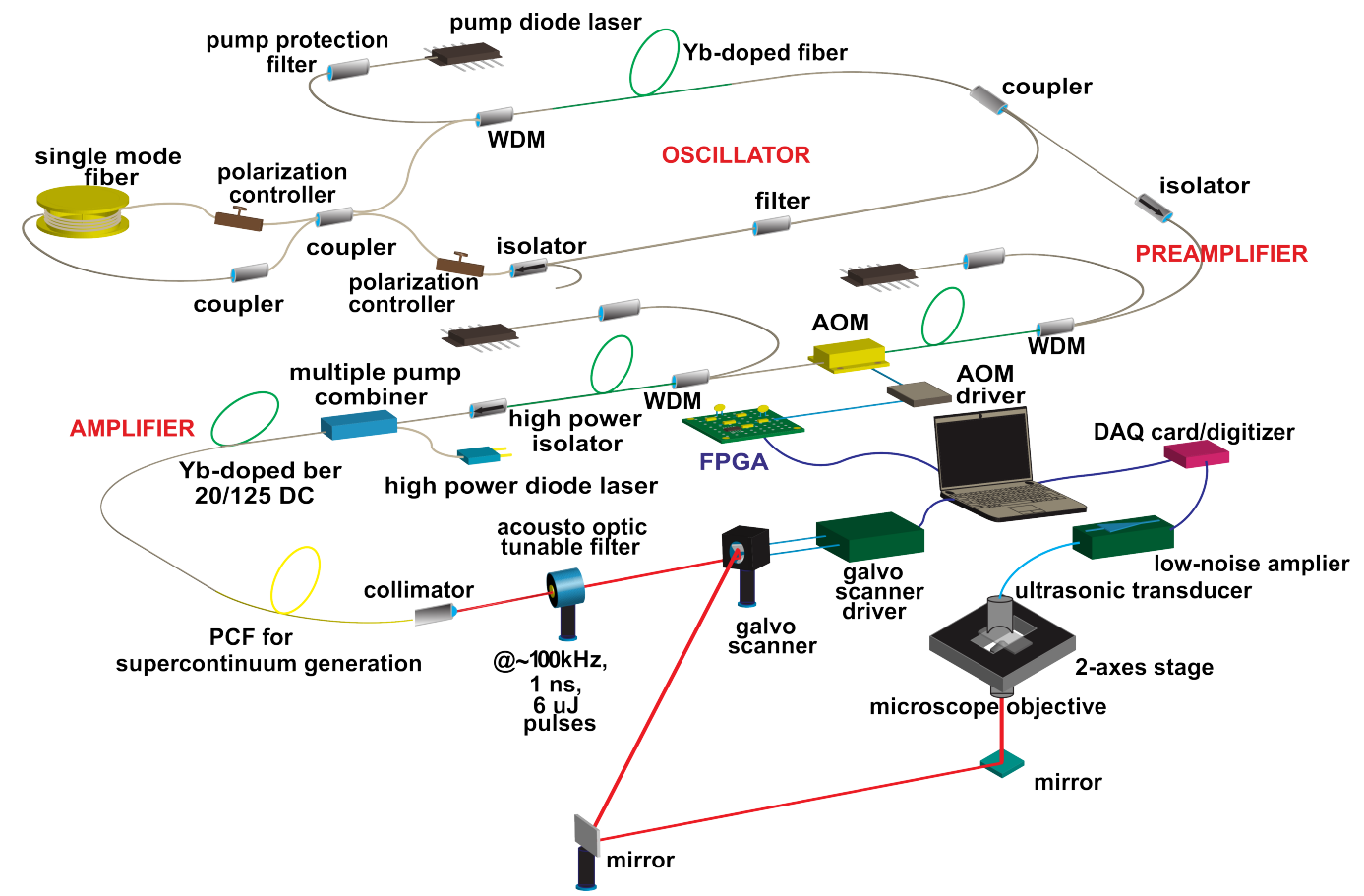

Figure 1. General schematic of laser and photoacoustic microscopy system

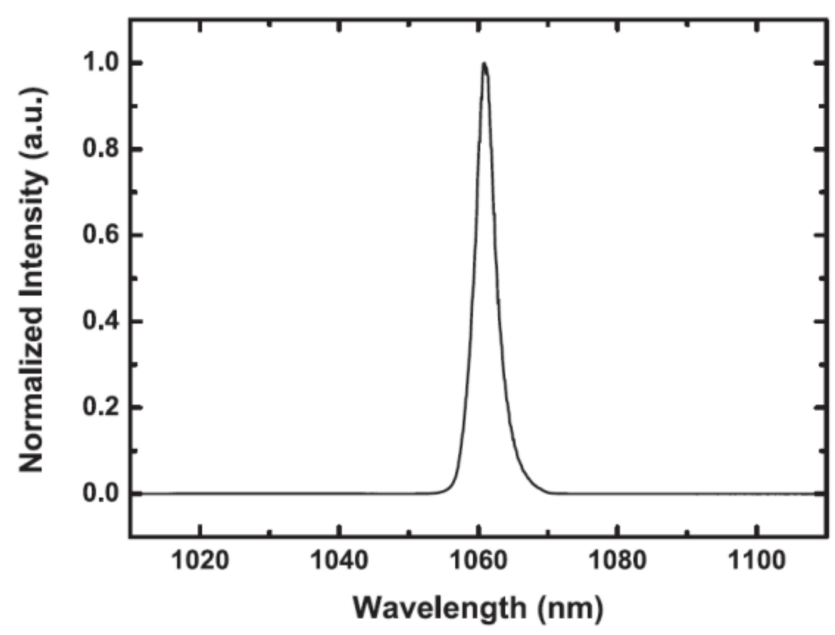

Figure 2. Infrared spectrum before supercontinuum 


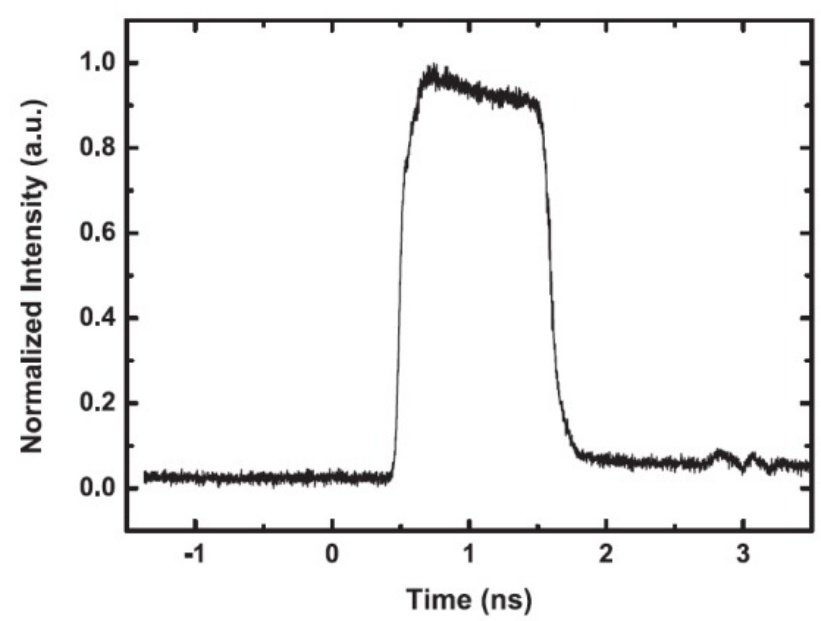

Figure 3. Temporal profile of laser

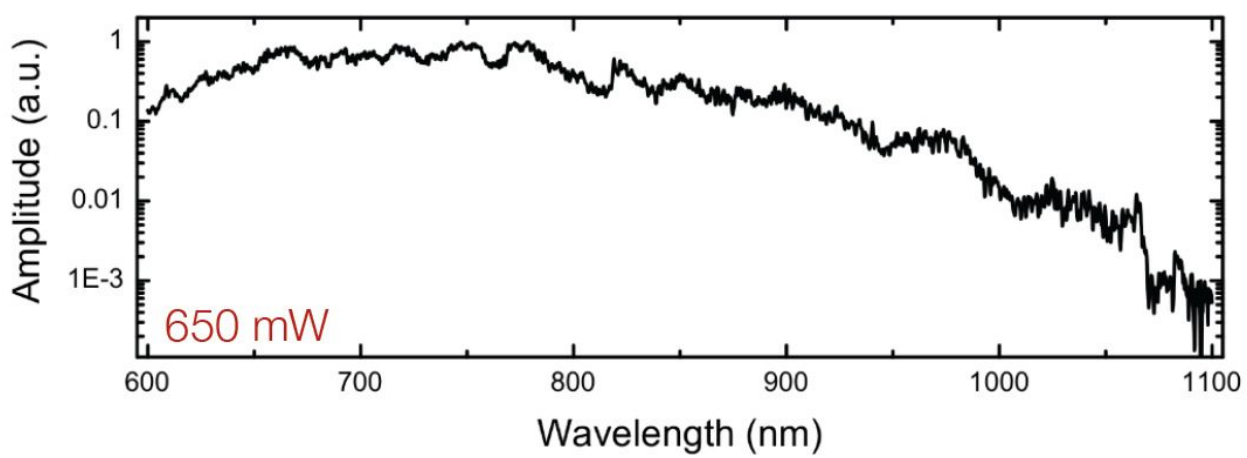

Figure 4. Spectrum of supercontinuum output 